\title{
Heritability of Intracortical Inhibition and Facilitation
}

\author{
Maria Concetta Pellicciari, ${ }^{1}$ Domenica Veniero, ${ }^{1,2}$ Cristina Marzano, ${ }^{3}$ Fabio Moroni, ${ }^{3}$ Cornelia Pirulli, ${ }^{2}$ \\ Giuseppe Curcio, ${ }^{4}$ Michele Ferrara, ${ }^{4}$ Carlo Miniussi, ${ }^{1,2}$ Paolo Maria Rossini, ${ }^{5,6,7}$ and Luigi De Gennaro ${ }^{1,3}$ \\ ${ }^{1}$ Istituto di Ricovero e Cura a Carattere Scientifico (IRCCS) San Giovanni di Dio, Fatebenefratelli, Brescia 25125, Italy, ${ }^{2}$ Department of Biomedical Science \\ and Biotechnology, University of Brescia, Brescia 25121, Italy, ${ }^{3}$ Department of Psychology, University of Rome "Sapienza," Rome 00185, Italy, ${ }^{4}$ Department \\ of Health Sciences, University of L'Aquila, L’Aquila 67100, Italy, ${ }^{5}$ IRCCS e Casa di Cura San Raffaele Rome, Rome 00187, Italy, ${ }^{6}$ IRCCS e Casa di Cura San \\ Raffaele Cassino, Cassino 03043, Italy, and ${ }^{7}$ Neurology Clinic, University Campus Biomedico, Rome 00155, Italy
}

The present twin study investigates heritability of motor cortex excitability, measured by the paired pulse transcranial magnetic stimulation technique. Specifically, intracortical facilitation (ICF) and inhibition (ICI) and corticospinal excitability were tested in monozygotic (MZ), dizygotic (DZ), and unrelated pairs (UP). Robust ICF and ICI effects were found, with a higher similarity of MZ than DZ and UP pairs. Heritability estimates $\left(h^{2}\right)$ were 0.80 for ICI and 0.92 for ICF. However, corticospinal excitability did not show significant differences between MZ and DZ pairs, whereas both significantly differed from UP. Hence, the study provides—for the first time-a clear evidence of heritable individual differences in motor cortex excitability.

\section{Introduction}

Evidence is accumulating that brain structure and function are under considerable genetic influence. The genetic contribution to several cognitive skills, such as verbal and spatial abilities, perceptual speed, and intelligence is now indisputable (Boomsma et al., 2002). Neuroimaging studies also indicate a relevant genetic influence on global and regional brain structure (Peper et al., 2007), morphologic similarities in the brain of monozygotic (MZ) twins (Wright et al., 2002) and specific variations in total gray and white matter volume (Baaré et al., 2001). Moreover, electrophysiological studies point to the heritability of bioelectrical cortical activity across different circadian phases, both in waking (van Beijsterveldt and van Baal, 2002) and sleep (De Gennaro et al., 2008).

However, little is known about genetic influences on cortical excitability in humans. Transcranial magnetic stimulation (TMS) is a well established method to noninvasively measure the threshold for cortical excitability, and the paired-pulse (PP) TMS protocol is one of the most consolidated, consisting of a subthreshold conditioning stimulus followed-after a given interval-by suprathreshold test stimulus (where "threshold" means the intensity of stimulation for eliciting $50 \%$ of motor responses in a series of 10 stimuli) delivered to the same cortical area ( $\mathrm{Ku}$ jirai et al., 1993). Amplitudes of responses are inhibited [intracortical inhibition (ICI)] with short interstimulus intervals [1 to $5 \mathrm{~ms}$ interstimulus intervals (ISIs)] and facilitated [intracortical facilitation (ICF)] with longer interstimulus intervals (Kujirai et

Received May 5, 2009; accepted June 5, 2009.

This work was supported by the Ministero dell'Università e della Ricerca (PRIN 2006-2006062871 to L.D.G.). L.D.G., C.M., M.F., and P.M.R. designed the research. M.C.P., D.V., C.M., F.M., C.P., and G.C. performed all experiments. Statistical analyses were done by L.D.G. and D.V. All authors discussed the results and commented on this manuscript. L.D.G, M.C.P., P.M.R., and D.V. wrote this manuscript.

Correspondence should be addressed to Luigi De Gennaro, Department of Psychology, Section of Neuroscience, University of Rome "Sapienza," Via dei Marsi 78, Rome 00185, Italy. E-mail: luigi.degennaro@uniroma1.it.

DOI:10.1523/JNEUROSCI.2112-09.2009

Copyright $\odot 2009$ Society for Neuroscience $\quad$ 0270-6474/09/298897-04\$15.00/0 al., 1993). In such a way, PP technique allows an evaluation of both inhibitory and excitatory neuronal circuits in the motor cortex (Ziemann et al., 1998). To date, there are no studies evaluating whether genetic heterogeneity contributes to the high interindividual variability of cortical excitability threshold levels, compared with the intersession-i.e., intraindividual-variability (Boroojerdi et al., 2000; Maeda et al., 2002; Wassermann, 2002; Orth et al., 2003). The basis of this phenotypic variability is not fully understood yet, although it is assumed that much of the difference among individuals and many of the quantitative traits are underpinned by genetic differences in gene expression (Myers et al., 2007); so far, few studies have directly evaluated the relationship between genetic factors and motor cortex activation, in relation to specific polymorphisms (Kleim et al., 2006; Cheeran et al., 2008) and genetic defects (Schwenkreis et al., 2002).

A well established approach to investigate phenotypic variability is the comparison between $\mathrm{MZ}$ and dizygotic (DZ) twins (Boomsma et al., 2002). Twin studies allow discriminating between environment and genetic effects, based on the assumption that $\mathrm{MZ}$ twins have identical genotypes, whereas DZ twins on average share only $50 \%$ of their genotype. Hence, the present study aimed to investigate the presence and amount of heritability of motor cortex excitability by means of a PP-TMS protocol in $\mathrm{MZ}$ and DZ twin pairs compared with a control group of unrelated pairs (UP).

\section{Materials and Methods}

Subjects. Fifty-two right-handed healthy volunteers [16 MZ twins $($ age $=24.9 \pm 2.6$ years $), 16 \mathrm{DZ}$ twins $($ age $=24.3 \pm 2.4$ years $)$, and 20 UP subjects (age $=24.2 \pm 0.7$ years) ] were selected from a university student population. The absence of epilepsy and any other medical or psychiatric disorders were inclusion criteria. The female pairs (four $\mathrm{MZ}$, three DZ, and five UP) were studied during the follicular phase of their menstrual cycle.

Zygosity was determined by a standardized questionnaire and morphological examination and confirmed by a DNA-based analysis. DNA was extracted from cheek cells collected using a sponge-tipped swab. 
Homozygosity was determined by using six highly polymorphic short tandem repeat loci. With these markers, the probability that any twin pair was MZ if all markers were concordant was $99.9 \%$. The UP group was formed from the whole sample of subjects who volunteered to other TMS studies, by randomly selecting each person from subgroups homogeneous for gender and age. The subjects gave their written informed consent; the protocol of the study was approved by the local Institutional Ethics Committee and conducted in accordance with the Declaration of Helsinki.

Transcranial magnetic stimulation. Motor cortex stimulation was performed via a Magstim 200 Mono Pulse connected to a Bistim module and to a figure-of-eight (double $70 \mathrm{~mm}$ ) coil (Magstim Company), the peak magnetic field being 2.2 Tesla. The motor-evoked potentials (MEPs) of the hand muscle were recorded from the abductor digiti minimi (ADM). Two $\mathrm{Ag}-\mathrm{AgCl}$ surface cup electrodes of $9 \mathrm{~mm}$ diameter were used: the active electrode over the muscle belly and the reference electrode over the metacarpophalangeal joint of the little finger (skin/electrode impedance, $<10 \mathrm{~K} \Omega$ ). MEPs were stored and analyzed by an EMG-dedicated software (Myto, EBNeuro).

Experimental design. Subjects were seated on a comfortable chair in a soundproof room and tested at the same time of day (10:30 P.M.).

The most effective point on the subject's scalp for eliciting an ADM response was localized by positioning the coil such that the junction region of the figure-of-eight coil was approximately over the central sulcus and moving the coil in $1 \mathrm{~cm}$ steps. The coil was positioned tangentially to the scalp and pointing in the anteromedial direction, $45^{\circ}$ from the midsagittal axis of the subject's head, and the handle of the coil posteriorly oriented. In this way, the induced current in the brain flowed nearly perpendicular to the orientation of the central sulcus, and the lowest motor thresholds (MTs) were defined according to international standards (Maeda et al., 2002).

The observer bias has been partially controlled by using a different experimenter for each member of the pairs. However, the experimenters were not completely blind toward the information to which group (MZ, $\mathrm{DZ}, \mathrm{UP})$ the individual belonged.

Motor thresholds. With the muscle relaxed and monitored by EMG and visual feedback, MT was measured according to international standards as the lowest intensity level of stimulation able to produce at least five MEPs with $100 \mu \mathrm{V}$ of amplitude (peak-to-peak) in 10 consecutive stimulations (Rossini et al., 1994). After MT, two further points on the intensity scale were defined, by adapting the criteria proposed by Mills and Nithi (1997). First, the maximum intensity at which 10 stimuli all produced no response was found by decreasing intensity in $1 \%$ steps; this was defined as the lower threshold (LT). Second, the minimum intensity at which 10 stimuli all produced a positive response was found by increasing the intensity in $1 \%$ steps from the lowest level, which so far had not resulted in a "no response"; this was defined as the upper threshold (UT).

Intracortical paired pulses. According to the intracortical PP protocol (Kujirai et al., 1993), two stimuli were delivered in close sequence to the left motor cortex through a single stimulating coil. Thus, the modulatory effect of the conditioning stimulus on the motor response (ADM) elicited by the test stimulus was assessed. ISIs between the conditioning and test pulses were as follows: $1,3,7,12 \mathrm{~ms}$, the first two and the last two being expected to produce ICI and ICF, respectively. The intensity of the conditioning shock was set at $70 \%$ of the individual MT at muscle rest, whereas the test one was set at $130 \%$. Eight responses per condition were collected. The peak-to-peak amplitude to each pulse was measured and subsequently averaged off-line.

The baseline level of MEP responses (unconditioned responses) was measured with an independent series of 12 test stimuli, administered alone at $130 \%$ of the individual motor threshold (test alone). Hence, each intracortical PP session included five blocks: test alone, 1, 3, 7, $12 \mathrm{~ms}$.

Data analysis. MTs were compared by one-way ANOVAs comparing the three groups (MZ, DZ, UP). Dependent variables of these ANOVAs were MT, LT, and UT values.

Single MEP amplitudes were measured peak-to-peak between the two largest peaks of opposite polarity. Changes in MEP amplitude as a consequence of conditioning pulse administration were expressed in terms
Table 1. $r_{\text {Icc }}$ of corticospinal excitability

\begin{tabular}{|c|c|c|c|c|c|c|c|c|c|}
\hline & \multirow[b]{2}{*}{$M Z$} & \multirow[b]{2}{*}{ DZ } & \multirow[b]{2}{*}{ UP } & \multicolumn{2}{|c|}{$\begin{array}{l}\text { MZ versus } \\
\text { DZ }\end{array}$} & \multicolumn{2}{|c|}{$\begin{array}{l}\text { MZ versus } \\
\text { UP }\end{array}$} & \multicolumn{2}{|c|}{$\begin{array}{l}\text { DZ versus } \\
\text { UP }\end{array}$} \\
\hline & & & & Z & $p$ & Z & $p$ & Z & $p$ \\
\hline & $r_{\text {ICC }}=0.79$ & $r_{\text {ICC }}=0.69$ & $r_{\text {ICC }}=-0.08$ & 0.35 & 0.36 & 1.98 & 0.02 & 1.58 & 0.06 \\
\hline & $r_{\mathrm{ICC}}=0.72$ & $r_{\mathrm{ICC}}=0.70$ & $r_{\text {ICC }}=-0.04$ & 0.06 & 0.47 & 1.62 & 0.05 & 1.55 & 0.04 \\
\hline UT & $r_{\text {ICC }}=0.65$ & $r_{\mathrm{ICC}}=0.79$ & $r_{\text {ICC }}=-0.03$ & -0.47 & 0.32 & 1.37 & 0.08 & 1.88 & 0.03 \\
\hline
\end{tabular}

$r_{\text {ICc }}$ of corticospinal measures for LT, UT, and standard MT, within MZ and DZ twin pairs and UP. Results of the significance testing on the difference between $r_{\mathrm{ICC}}$ of $\mathrm{MZ}, \mathrm{DZ}$, and $\mathrm{UP}$ are also reported ( $p \leq 0.05$, uncorrected for multiple comparisons)

of the ratio between conditioned (preceded by conditioning pulse) and unconditioned responses.

Changes in MEP amplitude were compared by a two-way $3 \times 4$ mixed design ANOVA, Group (MZ, DZ, UP) $\times$ ISI (1, 3, 7, $12 \mathrm{~ms})$, with the within-subject factor ISI.

The degree of within-pairs similarity has been evaluated by means of intraclass correlation coefficients $\left(r_{\text {ICC }}\right)$ on MTs and changes in MEP amplitude, separately calculated for each group (Shrout and Fleiss, 1979). Then, the significance testing of the difference between these $r_{\text {ICC }}$ was performed after a $r$-to- $z$ transform (Griffin and Gonzalez, 1995), and a one-tailed probability of difference between $r_{\text {ICC }}$ values was considered, since we have a clear a priori hypothesis on the direction of expected differences based on the assumptions of twin studies.

We set the significance levels for the ANOVAs to $p \leq 0.01$ to correct for multiple comparisons, whereas it was set to $p \leq 0.05$ for the $t$ and $Z$ tests. All data are given as means \pm SE.

\section{Results}

A high phenotypic variability for both motor thresholds and cortical excitability parameters was confirmed in the sample population independently from subjects' belonging to a specific zygosity group. In fact, ANOVAs on these measures showed a robust interindividual variability of the whole sample, with a significant effect for the factor "subjects" (MTs: $F=14.13, p<$ 0.0001; ICI and ICF: $F=3.62, p<0.0001$ ).

\section{Motor thresholds}

Comparison of MTs in the three groups did not show significant differences $\left(\mathrm{LT}: F_{(2,49)}=0.63, p=0.54\right.$; MT: $F_{(2,49)}=0.42, p=$ 0.66 ; UT: $\left.F_{(2,49)}=0.29, p=0.75\right)$. However, the three groups were different with respect to their within-pair similarity. Table 1 reports the results of the intraclass correlations for any measure of MTs. The coefficients were quite high within twin pairs and pointed to a significant similarity for both MZ and DZ pairs, whereas a low similarity was found within UP pairs. As a consequence, differences between $\mathrm{MZ}$ and $\mathrm{DZ}$ correlations were not significant, and differences between both $M Z$ and DZ versus UP correlations were mostly significant (Table 1).

\section{Intracortical excitability}

As detailed in Figure 1-showing MEP amplitude changes of MZ, DZ, and UP pairs across the range of ISIs considered-a clear inhibition (ICI) was observed at 1 and $3 \mathrm{~ms}$, whereas ICF was evident at 7 and $12 \mathrm{~ms}$. Regardless of group, a cortical inhibition was found at $1 \mathrm{~ms}$ (indicated by the $74.9 \%$ MEP decrease) and at $3 \mathrm{~ms}$ (50.3\% MEP amplitude decrease). The cortical facilitation was expressed by a $28.8 \%$ MEP increase at $7 \mathrm{~ms}$ and a $46.8 \%$ increase at $12 \mathrm{~ms}$.

The Group $\times$ ISI ANOVA on MEP amplitude changes neither showed main effect for Group $\left(F_{(2,49)}=0.03 ; p=0.96\right)$, nor for Group $\times$ ISI interaction $\left(F_{(6,147)}=0.88 ; p=0.51\right)$. The effect for ISI was significant $\left(F_{(3,147)}=78.76 ; p<1^{-8}\right)$, and post hoc comparisons pointed to significant differences between each ISI (ISI 1 


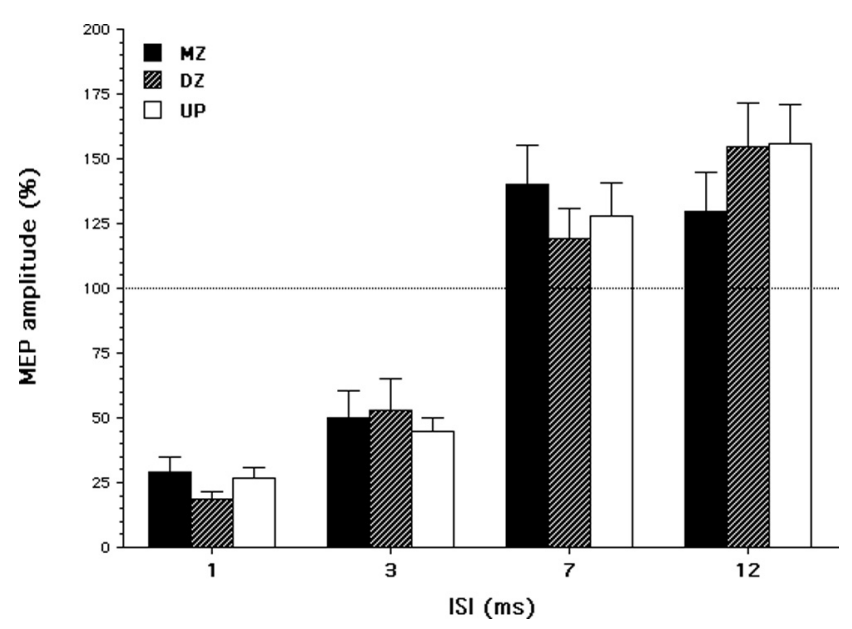

Figure 1. Mean ( $\pm S E$ ) values of intracortical excitability in $16 \mathrm{MZ}, 16 \mathrm{DZ}$ twins, and $20 \mathrm{UP}$ subjects measured by the PP TMS technique. Mean changes in MEP amplitude expressed as the ratio between conditioned responses at different ISI divided by test (unconditioned) responses. Horizontal line indicates MEP amplitude at baseline. Error bars, SEM.

Table 2. $r_{\text {Icc }}$ of intracortical excitability

\begin{tabular}{|c|c|c|c|c|c|c|c|c|c|}
\hline & \multirow[b]{2}{*}{ MZ } & \multirow[b]{2}{*}{ DZ } & \multirow[b]{2}{*}{ UP } & \multicolumn{2}{|c|}{$\begin{array}{l}\text { MZ versus } \\
\text { DZ }\end{array}$} & \multicolumn{2}{|c|}{$\begin{array}{l}\text { MZ versus } \\
\text { UP }\end{array}$} & \multicolumn{2}{|c|}{$\begin{array}{l}\text { DZ versus } \\
\text { UP }\end{array}$} \\
\hline & & & & Z & $p$ & Z & $p$ & Z & $p$ \\
\hline $1 \mathrm{~ms}$ & $r_{\text {ICC }}=0.37$ & $r_{\text {ICC }}=0.25$ & $r_{\text {ICC }}=0.44$ & 0.21 & 0.42 & -0.14 & 0.44 & -0.37 & 0.36 \\
\hline $3 \mathrm{~ms}$ & $r_{\text {ICC }}=0.78$ & $r_{\mathrm{ICC}}=0.11$ & $r_{\text {ICC }}=-0.19$ & 1.48 & 0.07 & 2.11 & 0.02 & 0.52 & 0.30 \\
\hline $7 \mathrm{~ms}$ & $r_{\mathrm{ICC}}=0.87$ & $r_{\mathrm{ICC}}=0.25$ & $r_{\text {ICC }}=0.35$ & 1.70 & 0.04 & 1.65 & 0.05 & -0.19 & 0.42 \\
\hline $12 \mathrm{~ms}$ & $r_{\text {ICC }}=0.62$ & $r_{\text {ICC }}=0.31$ & $r_{\text {ICC }}=-0.01$ & 0.64 & 0.26 & 1.25 & 0.10 & 0.56 & 0.29 \\
\hline
\end{tabular}

$r_{\text {ICc }}$ for the intracortical excitability values, measured by the PP technique, within $\mathrm{MZ}$ and $\mathrm{DZ}$ twin pairs and UP. Results of the significance testing on the difference between $r_{\text {ICC }}$ of MZ, DZ, and UP are also reported ( $p \leq 0.05$, uncorrected for multiple comparisons).

ms vs ISI $3 \mathrm{~ms}, p=0.008$; ISI $1 \mathrm{~ms}$ vs ISI $7 \mathrm{~ms}$ and ISI $12 \mathrm{~ms}, p<$ $1^{-8}$; ISI $3 \mathrm{~ms}$ vs ISI $7 \mathrm{~ms}$ and ISI $12 \mathrm{~ms}, p<1^{-8}$ ), whereas the comparison between ISI $7 \mathrm{~ms}$ versus ISI $12 \mathrm{~ms}$ was close to significance ( $p=0.07)$. Therefore, clear effects of cortical inhibition and facilitation were found without any significant difference between groups.

Results of the intraclass correlations are reported in Table 2 and point to a difference between similarity within $\mathrm{MZ}$ compared with DZ and UP pairs. Notwithstanding the small sample size for this kind of analysis, $r_{\text {ICC }}$ coefficients of MZ compared with DZ and UP pairs were different for the $7 \mathrm{~ms}$ ISI and approached significance for the $3 \mathrm{~ms}$ ISI. However, comparisons between DZ and UP pairs never reached significance.

For the analysis of mean similarity, the four ISIs were grouped into two groups on the basis of known effects. Therefore, the mean similarity for ICI identified at short ISIs (1 and $3 \mathrm{~ms}$ ) was 0.58 within $\mathrm{MZ}, 0.18$ within $\mathrm{DZ}$, and 0.12 within UP, whereas mean similarity for ICF at longer ISIs (7 and $12 \mathrm{~ms}$ ) was 0.74 within $\mathrm{MZ}, 0.28$ within $\mathrm{DZ}$, and 0.17 within UP.

Estimates of broad-sense heritability were calculated on these values according to the classical approach (Falconer and Mackay, 1996) by the Falconer's method $\left[h^{2}=2\left(r_{\mathrm{MZ}}-r_{\mathrm{DZ}}\right)\right]$. This model assumes independence between the environment and genetic factors, and heritability values range from 0 (no genetic contribution) to 1 (complete genetic contribution). Thus, the observed similarities in twins correspond to a heritability estimate of $h^{2}=0.80$ for cortical inhibition and of $h^{2}=0.92$ for cortical facilitation.

\section{Discussion}

The classical PP-TMS protocol as originally described (Kujirai et al., 1993) has been widely used to explore the state of activation of distinct inhibitory and excitatory intracortical circuits in different physiological and pathological conditions. However, the genetic contribution on ICI and ICF has never been investigated so far. To assess this issue, a twin study, which provides an approach to investigate genetic and environmental contributions to phenotypic variability, has been carried out. Our results point to a heritability estimate of 0.80 for cortical inhibition and 0.92 for facilitation, suggesting that individual variability in $\mathrm{PP}$ responses reflects individual genotype differences.

A major influence of genes on human brain volume and surface morphology, mostly for the sensorimotor areas, has been demonstrated by twin studies (Peper et al., 2007). Current results extend the influence of genes also to functional parameters or features rather than morphology/anatomy, suggesting that a significant proportion of variability in the individual responses to PP protocol could be attributable to biological differences between subjects, such as different endophenotypes (Wassermann, 2002). In the same vein, a high interindividual variability associated to a relatively high intraindividual stability in motor cortical plasticity, as assessed by paired associative stimulation (PAS) (Fratello et al., 2006) can be explained by the genetic determination of some PAS-induced excitability changes (Missitzi et al., 2008).

Moreover, present results are coherent with studies investigating how individual genetic characteristics modulate human response to TMS both in normal and pathological conditions (Kleim et al., 2006; Cheeran et al., 2008). Interestingly, we have shown heritability of both ICF and ICI effects, whereas different genetic subtypes of ataxia only differed for changes in intracortical facilitation (Schwenkreis et al., 2002).

A different picture emerges when examining the within-pair similarity for measures of corticospinal excitability: a high similarity within both twin groups coexists with a lack of between-group differences; however, the UP group shows a very low similarity, at a significant difference from both twin groups. It should be remembered that MTs reflect the excitability of both primary motor cortex and corticospinal tract (Reid et al., 2002). Hence, the lack of significant heritability, as indicated by the similarity within MZ and DZ groups, should reasonably be attributable to intrinsic characteristics of subcortical, spinal, and peripheral relays and fibers. This interpretation is coherent with the significantly different within-pair similarity of the UP group. An indirect and partial support comes from the absence of any genetic influence on the conduction velocity of impulse propagation along peripheral nerves (Rijsdijk and Boomsma, 1997).

Some limitations, however, characterize the current study, since (1) the statistical power is relatively low because of the intrinsic peculiarity of twin designs, which restricts to eight observations the final size considered for the estimates of withinpair similarity by the intraclass correlations; (2) height and arm length have not been matched within UP subjects, preventing to control for a possible effect of these specific unshared anatomical factors on the motor thresholds; and (3) ICI and ICF have been tested with a single intensity of the conditioning pulse $(70 \%$ of MT), which induces strong ICI in most subjects, and floor effects cannot be excluded (Rosenkranz et al., 2007). Finally, although the classical model of twin studies is based on the comparison of phenotypic variability between $\mathrm{MZ}$ and $\mathrm{DZ}$ twins, and the assumption of shared environment is not tenable for UP subjects, 
nevertheless, one would expect intraclass correlation coefficients to show a progressively decreasing trend $(\mathrm{MZ}>\mathrm{DZ}>\mathrm{UP})$. We think that the lack of differences between DZ and UP groups should be explained in terms of different sources of variability attributable to unshared environmental factors and to error variability in our relatively small number of pairs. Future studies with larger samples and matching unrelated pairs for their height and arm length will elucidate this issue.

In conclusion, this study provides the first evidence for heritable individual differences in the neural substrates of motor cortex and corticospinal tract excitability. Although preliminary, the present finding could shed light on mechanisms by which genes influence normal and abnormal brain excitability (Buhmann et al., 2004).

\section{References}

Baaré WF, van Oel CJ, Hulshoff Pol HE, Schnack HG, Durston S, Sitskoorn MM, Kahn RS (2001) Volumes of brain structures in twins discordant for schizophrenia. Arch Gen Psychiatry 58:33-40.

Boomsma D, Busjahn A, Peltonen L (2002) Classical twin studies and beyond. Nat Rev Genet 3:872-882.

Boroojerdi B, Kopylev L, Battaglia F, Facchini S, Ziemann U, Muellbacher W, Cohen LG (2000) Reproducibility of intracortical inhibition and facilitation using the paired-pulse paradigm. Muscle Nerve 23:1594-1597.

Buhmann C, Gorsler A, Bäumer T, Hidding U, Demiralay C, Hinkelmann K, Weiller C, Siebner HR, Münchau A (2004) Abnormal excitability of premotor-motor connections in de novo Parkinson's disease. Brain 127:2732-2746.

Cheeran B, Talelli P, Mori F, Koch G, Suppa A, Edwards M, Houlden H, Bhatia K, Greenwood R, Rothwell JC (2008) A common polymorphism in the brain derived neurotrophic factor gene (BDNF) modulates human cortical plasticity and the response to rTMS. J Physiol 586:5717-5725.

De Gennaro L, Marzano C, Fratello F, Moroni F, Pellicciari MC, Ferlazzo F, Costa S, Couyoumdjian A, Curcio G, Sforza E, Malafosse A, Finelli LA, Pasqualetti P, Ferrara M, Bertini M, Rossini PM (2008) The electroencephalographic fingerprint of sleep is genetically determined: a twin study. Ann Neurol 64:455-460.

Falconer DS, Mackay TFC (1996) Introduction to quantitative genetics. Ed 4. Harlow, Essex, UK: Addison Wesley Longman.

Fratello F, Veniero D, Curcio G, Ferrara M, Marzano C, Moroni F, Pellicciari MC, Bertini M, Rossini PM, De Gennaro L (2006) Modulation of corticospinal excitability by paired associative stimulation: reproducibility of effects and intraindividual reliability. Clin Neurophysiol 117:2667-2674.

Griffin D, Gonzalez R (1995) Correlational analysis of dyad-level data in the exchangeable case. Psychol Bull 118:430-439.

Kleim JA, Chan S, Pringle E, Schallert K, Procaccio V, Jimenez R, Cramer SC (2006) BDNF val66met polymorphism is associated with modified experience-dependent plasticity in human motor cortex. Nat Neurosci 9:735-737.

Kujirai T, Caramia MD, Rothwell JC, Day BL, Thompson PD, Ferbert A,
Wroe S, Asselman P, Marsden CD (1993) Corticocortical inhibition in human motor cortex. J Physiol 471:501-519.

Maeda F, Gangitano M, Thall M, Pascual-Leone A (2002) Inter- and intraindividual variability of paired-pulse curves with transcranial magnetic stimulation (TMS). Clin Neurophysiol 113:376-382.

Mills KR, Nithi KA (1997) Corticomotor threshold to magnetic stimulation: normal values and repeatability. Muscle Nerve 20:570-576.

Missitzi J, Gentner R, Geladas N, Classen J, Klissouras V (2008) Genetic variation of plasticity in human motor cortex. Brain Stim 1:299.

Myers AJ, Gibbs JR, Webster JA, Rohrer K, Zhao A, Marlowe L, Kaleem M, Leung D, Bryden L, Nath P, Zismann VL, Joshipura K, Huentelman MJ, Hu-Lince D, Coon KD, Craig DW, Pearson JV, Holmans P, Heward CB, Reiman EM, Stephan D, Hardy J (2007) A survey of genetic human cortical gene expression. Nat Genet 39:1494-1499.

Orth M, Snijders AH, Rothwell JC (2003) The variability of intracortical inhibition and facilitation. Clin Neurophysiol 114:2362-2369.

Peper JS, Brouwer RM, Boomsma DI, Kahn RS, Hulshoff Pol HE (2007) Genetic influences on human brain structure: a review of brain imaging studies in twins. Hum Brain Mapp 28:464-473.

Reid A, Chiappa K, Cros D (2002) Motor threshold, facilitation and silent period in cortical magnetic stimulation. In: Handbook of transcranial magnetic stimulation (Pascual-Leone A, Dvey NJ, Rothwell J, Wassermann EM, Puri BK, eds), pp 97-112. London: Arnold.

Rijsdijk FV, Boomsma DI (1997) Genetic mediation of the correlation between peripheral nerve conduction velocity and IQ. Behav Genet 27:87-98.

Rosenkranz K, Kacar A, Rothwell JC (2007) Differential modulation of motor cortical plasticity and excitability in early and late phases of human motor learning. J Neurosci 27:12058-12066

Rossini PM, Barker AT, Berardelli A, Caramia MD, Caruso G, Cracco RQ, Dimitrijević MR, Hallett M, Katayama Y, Lücking CH (1994) Noninvasive electrical and magnetic stimulation of the brain, spinal cord and roots: basic principles and procedures for routine clinical application. Report of an IFCN committee. Electroencephalogr Clin Neurophysiol 91:79-92.

Schwenkreis P, Tegenthoff M, Witscher K, Börnke C, Przuntek H, Malin JP, Schöls L (2002) Motor cortex activation by transcranial magnetic stimulation in ataxia patients depends on the genetic defect. Brain 125:301-309.

Shrout PE, Fleiss JL (1979) Intraclass correlations: uses in assessing rater reliability. Psychol Bull 86:420-428.

van Beijsterveldt CE, van Baal GC (2002) Twin and family studies of the human electroencephalogram: a review and a meta-analysis. Biol Psychol 61:111-138.

Wassermann EM (2002) Variation in the response to transcranial magnetic brain stimulation in the general population. Clin Neurophysiol 113:1165-1171.

Wright IC, Sham P, Murray RM, Weinberger DR, Bullmore ET (2002) Genetic contributions to regional variability in human brain structure: methods and preliminary results. Neuroimage 17:256-271.

Ziemann U, Chen R, Cohen LG, Hallett M (1998) Dextromethorphan decreases the excitability of the human motor cortex. Neurology 51:13201324. 